\title{
CHEMICAL CONTENT OF NINE F-SUPERGIANTS FROM DIFFERENT REGIONS OF THE LMC
}

\author{
V. HILL \\ DASGAL/URA 335 du C.N.R.S. - Observatoire de Paris-Meudon, F-92195 \\ Meudon Cedex - E-mail: hill@memaga.obspm.fr
}

In order to extend the available abundance information in the Magellanic Clouds, and particularly the elemental ratios, we have undertaken a homogenous sudy of supergiants stars in both Clouds using high resolution spectroscopy. We compare the abundance ratios obtained for nine LMC F-supergiants (Hill et al., 1994) with those for three SMC similar stars (Spite et al., 1989). Let us note in particular that:

1. Although the nine stars are located in different regions of the LMC, we found a surprisingly uniform iron abundance for all stars $([\mathrm{Fe} / \mathrm{H}]=-0.27 \pm 0.07$ dex $)$ and this small scatter in abundance (small compared with the intrisic error) holds for most other elements. This is remarkable and tends to show that the ISM giving birth to these stars was very homogeneous, which in turns, means that the mixing in the LMC is very efficient.

2. The carbon abundance $[\mathrm{C} / \mathrm{H}]$ in the stars is found to exceed the HII regions observed values by up to 0.5 dex. To discuss this difference, we used $[\mathrm{C} / \mathrm{S}]$ ratios, available both in the supergiants and the HII regions. In Canopus, the difference with respect to Galactic HII regions is considered to come from the mixing experienced by the star during the 1st dredge-up phase. But in the Clouds, if we correct the supergiants' carbon abundance for mixing (assuming that the mixing is the same, i.e. the stars are carbon-depleted by $\approx 0.3$ dex), the discrepancy (in [C/S] values) still remains in the LMC (+0.3 dex) and even more drastically in the SMC (+0.5 dex). So, mixing cannot account for such a difference !

3. Oxygen in LMC supergiants is found to be depleted (mean $[\mathrm{O} / \mathrm{H}]=-0.35 \mathrm{dex}$ ) and similar to the HII regions abundances. [O/Fe] ratio has a small scatter in the LMC, as in the SMC. But in our galaxy, Canopus also shows a low oxygen abundance $(-0.24 \mathrm{dex})$ : is this a real effect (due to galactic evolution) or only due to a wrong analysis of supergiants' atmopheres ? The good concordance of HII regions and stars, plays in favour of a real effect. Then, one can consider the $[0 / \mathrm{Fe}]$ ratios to be the same in both clouds and in Canopus, in contrast with Galactic dwarfs for wich there is a nice $[\mathrm{O} / \mathrm{Fe}]$ over $[\mathrm{Fe} / \mathrm{H}]$ linear decrease. On the other hand, if there is a model atmosphere effect, one should look at the $\left([\mathrm{O} / \mathrm{Fe}]-[\mathrm{O} / \mathrm{Fe}]_{\text {canopus }}\right)$ ratios, and these behave like the Galactic dwarfs.

4. $s$ - and $r$-process elements heavier than barium show a tendency to be overabundant by a factor of +0.3 dex whereas there is no sign of anomaly for lighter elements $(\mathrm{Y}, \mathrm{Zr})$.

\section{References}

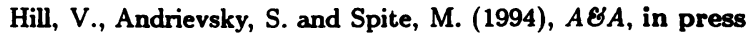

Spite, M., Barbuy, B. and Spite, F. (1989), $A \& A$, Vol. 222, pp. 35-40 